\section{EL CONSUMO DE INFORMACIÓN EN HUMANIDADES}

Julia Osca-Lluch, Ana Veyrat y Jesús Morales

Inst. de Historia de la Medicina y de la Ciencia López Piñero (UV-CSIC)

m.julia.osca.@uv.es; ana.veyrat@uv.es; jesus.morales@uv.es

Cómo citar este artículo/ Citation: Osca-Lluch, J.; Veyrat, A. y Morales, J. (2013). El consumo de información en Humanidades. Arbor, 189(760):a026. doi: http://dx.doi.org/10.3989/ arbor.2013.760n2012

Recibido: 5 enero 2010; Aceptado: 5 abril 2010.

RESUMEN: Se analizan los hábitos de publicación y de citación de los investigadores en ciencias humanas, a través de los trabajos publicados en una selección de revistas españolas de humanidades durante los años 2006 y 2007. El objetivo del estudio es conocer la tipología de los principales documentos que genera y consulta este colectivo de científicos en el desarrollo de sus investigaciones, así como identificar algunas de las características que definen su actividad científica. El estudio pone de manifiesto la importancia que para este colectivo de investigadores tienen los libros frente a los artículos de revistas, actas de congresos, artículos de prensa y actas de congresos, como documentos de publicación y de consulta.

PALABRAS CLAVE: Hábitos de citación; consumo de información; Humanidades; ciencias humanas; revistas científicas españolas.

\section{INTRODUCCIÓN}

La investigación científica solo tiene razón de ser desde el momento en que el autor comunica sus resultados al resto de la comunidad científica a través de una publicación con cierta capacidad de difusión $y$ al alcance de toda la comunidad investigadora interesada en dicho tema. Las publicaciones constituyen, por tanto, el producto final de la investigación. La mo-
Copyright: @ 2013 CSIC. Este es un artículo de acceso abierto distribuido bajo los términos de la licencia Creative Commons Attribution-Non Commercial (by-nc) Spain 3.0.

ABSTRACT: We analyze patterns of publication and citation of research in the humanities, through the work published in a selection of Spanish journals in the humanities during the years 2006 and 2007. The aim of this study was to determine the typology of the main documents produced and consulted by this group of scientists in the pursuit of their research, and to identify some of the defining characteristics of their scientific work. The study highlights the importance for this group of researchers of books rather journal articles, conference proceedings, newspaper articles and conference proceedings, as documents for publication and consultation.

KEYWORDS: Citation patterns; information consumption; Humanities; Spanish scientific journals.

derna normativa de la ciencia requiere que los científicos hagan referencia en sus trabajos a aquellos que han servido de base al suyo propio (Merton, 1977). Los trabajos que se publican en revistas científicas generalmente contienen citas bibliográficas que proporcionan los precedentes que existen sobre lo que el autor quiere exponer en su trabajo de investigación y 
posibilitan el establecimiento de relaciones entre los trabajos que reciben las citas y los que emiten referencias (trabajos fuente o citadores).

En los estudios bibliométricos se distingue con precisión entre citas (que una publicación recibe) y referencias (que una publicación hace de otras anteriores). Los estudios de las referencias bibliográficas, también denominados estudios del consumo de información, constituyen el medio idóneo de recuperación de información, ya que son la manifestación material del enlace que existe entre la labor de investigación que se expone y la labor de investigación precedente.

Las referencias proporcionan los precedentes sobre lo que los autores exponen en sus trabajos de investigación. Además nos informan del comportamiento y hábitos de los autores respecto a sus lecturas y pueden servir para racionalizar la alerta bibliográfica. Los estudios sobre el consumo de información a partir del análisis de las citas se construyen partiendo de esta premisa, pues proporcionan listas de publicaciones que han sido citadas, al tiempo que identificas las fuentes de las citaciones. A través del análisis de las referencias contenidas en los trabajos publicados se puede identificar el género documental de los documentos referenciados, el consumo de bibliografía nacional o extranjera, el núcleo de revistas más citadas, países de procedencia y el envejecimiento de la literatura científica (obsolescencia) medida a través de la vida media de las referencias bibliográficas y se pueden obtener diversos indicadores de actividad científica, entre los que destacan el índice de aislamiento (porcentaje de citas a publicaciones del propio país), el índice de autocitas (porcentaje de citas a la propia revista).

También, a través del estudio de las referencias, encontramos abundante información sobre los hábitos de consumo de las diversas comunidades científicas e indica qué autores, trabajos y temáticas son más relevantes. Los estudios bibliométricos sobre el consumo de información, basados en el análisis de referencias, demuestran que la información sobre una determinada disciplina se suele encontrar en un núcleo de revistas específicas, pero también en otras de temática general o incluso diferente que mantienen alguna relación con la disciplina en cuestión.

Las funciones que desempeñan las citas y referencias en el proceso de comunicación científica han sido ampliamente debatidas. De acuerdo con los trabajos de Kaplan (1965) y Merton (1969), la función principal de las referencias en la ciencia moderna es la d e reconocer que unos determinados datos, teorías, métodos, etc., proceden de los autores y publicaciones citados, en contraste con lo que sucedía en la ciencia antigua y tradicional, en la que servían casi exclusivamente para citar una autoridad clásica como funda- mento de una idea o doctrina. Para Burton y Kleber (1960), las referencias contenidas en los artículos publicados en un período concreto de tiempo y en una determinada área de trabajo, constituyen lo que se ha llamado "literatura activa circulante", es decir, la literatura que contiene la información viva que se esta utilizando en ese momento. Sobre esta base, los indicadores procedentes de las citas y referencias se apoyan en el supuesto de que los trabajos importantes son usualmente citados, mientras que los irrelevantes se ignoran. Sin embargo, la investigación sociológica en torno a las citas y referencias ha demostrado que la realidad es mucho más compleja y existen diferentes motivaciones a la hora de citar un trabajo. El propio Garfield (1979), creador de las bases de datos Journal Citation Index (JCR), ha puesto de relieve el hecho de que los autores o publicaciones extraordinariamente importantes tienden a darse por conocidos y a no ser citados explícitamente. Moravcsic y Murugesan (1975) demostraron que existe un elevado número de citas realizadas a la ligera, por formulismo o para salvar las apariencias. Otros autores han analizado la influencia de las barreras idiomáticas y nacionales, el peso de los diferentes tamaños de las áreas y subáreas científicas y de sus variados patrones en este terreno.

Aunque son muchos los estudios realizados sobre los hábitos de publicación de los investigadores de las distintas áreas tanto de la ciencia y la tecnología (Martín, 1999), de las ciencias médicas (López Piñero y Terrada, 1992;Lozano y Saez, 1999; Massó et al, 2001) y sociales (Ortega, 2004), ya sea en su conjunto o en el ámbito particular de los distintos campos o disciplinas científicas (Osca-Lluch et al., 1999), sin embargo, son menos frecuente los realizados en el campo de las humanidades. Los trabajos sobre el consumo de información han puesto de manifiesto la existencia de marcadas diferencias entre los hábitos de publicación de los diferentes científicos (Terrada et al., 1993). Uno de los aspectos que más diferencia a unas disciplinas de otras es el relativo al vehículo de difusión de los resultados de las investigaciones. Todos los estudios basados en los cómputos de citas coinciden en afirmar que en las ciencias experimentales la información se transmite principalmente a través de artículos de revista, que suman en ella porcentajes de citas superiores al 80 por ciento, seguidos de lejos por los libros, con tantos por ciento en torno a 10. Por el contrario, en las ciencias sociales y las humanidades y, por distintos motivos, también en las aplicaciones prácticas, predominan los libros, que reúnen entre el 50 y el 65 por ciento de las citas, en tanto que los artículos de revista tienen un peso relativamente modesto, que se manifiesta en porcentajes comprendidos entre el $10 \mathrm{y}$ el 35 (Broadus, 1971). Este distinto comportamiento a la hora de publicar tiene, por lo general, su correspondiente reflejo en las pautas de citación de los autores. 
De los trabajos realizados sobre los humanistas, se han obtenido unas características comunes a estos investigadores entre las que cabe mencionar que se observa que utilizan preferentemente los canales formales para actualizar su conocimiento, y dentro de ellos, suelen consultar más monografías que publicaciones periódicas. Los canales informales tienen una gran importancia, aunque los emplean fundamentalmente para mantener contactos personales con colegas, aunque tienen tendencia a trabajar de forma aislada, por lo que existen relativamente pocos grupos de colaboración en esta área y en general, tienen una gran capacidad idiomática, por lo que pueden hacer uso de documentos escritos en diferentes idiomas.

El objetivo de este trabajo es analizar el estudio de los hábitos de citación de los investigadores del área de humanidades, a través del estudio de las referencias bibliográficas de cinco revistas españolas muy representativas de esta área, así como identificar algunas de las características que definen su actividad científica.

\section{MATERIAL Y MÉTODOS}

Para este trabajo se ha decidido utilizar como unidad de análisis las referencias de los artículos publicados en las revistas Al-Qantara, Arbor, Dynamis, Hispania y Revista de Indias publicadas durante los años 2006 y 2007, que están indizadas en la base de datos Arts and Humanities Citation Index ( $\mathrm{AHCl}$ ), base de datos internacional elaborada por la empresa Thomson Reuters que se puede consultar a través del portal ISI Web of Knowledge.

Las revistas objeto de estudio son todas ellas publicaciones españolas, clasificadas en las bases de datos ISI en el área de humanidades:

Al-Qantara: revista de Estudios Árabes. (ISSN 02113589), inició su publicación en 1980, como continuación de Al-Andalus (1933-1978). Esta editada por el Instituto de Filología del Consejo Superior de Investigaciones Científicas. Está dedicada a la civilización del Islam clásico (hasta el siglo XVII incluido) con especial atención al Occidente islámico. Tiene una periodicidad semestral. Se publica en forma de dos fascículos anuales de unas 250 páginas cada uno.

Arbor, revista de Ciencia, Pensamiento y Cultura (ISSN 0210-1963), inició su publicación en 1944. Es una publicación periódica bimensual, editada por el Consejo Superior de Investigaciones Científicas. Se trata de una revista que tal como indican sus editores "se caracteriza por estar al servicio de la sociedad española y de la comunidad científica como instrumento de información, puesta al día, reflexión y debate".

Dynamis (0211-9536), inicio su publicación en 1981. Está editada por la Universidad de Granada. Está dedi- cada a la Historia de la Medicina y la Salud e Historia de las Ciencias. Tiene una periodicidad anual.

Hispania: revista española de historia (ISSN 00182141), inició su publicación en 1940. Está editada por el Instituto de Historia del Consejo Superior de Investigaciones Científicas. Se trata de una publicación, con periodicidad cuatrimestral, dedicada al estudio de las sociedades en las épocas medieval, moderna y contemporánea. Es considerada una de las revistas de historia general más significativas, considerada un excelente punto de referencia para observar la evolución del panorama historiográfico español (Tosete, 2002).

Revista de Indias (ISSN 0034-8341), inició su publicación en 1940. Tiene una periodicidad cuatrimestral. Está editada por el Instituto de Historia del Consejo Superior de Investigaciones Científicas. Con una larga y consolidada tradición Revista de Indias fue y continúa siendo un foro de debate de la historia de América. Las temáticas están abiertas a distintos aspectos como son los sociales, culturales, políticos y económicos, abarcando desde el mundo prehispánico a la actualidad de Iberoamérica.

Se han analizado todas las referencias bibliográficas contenidas en los documentos publicados por estas cinco revistas seleccionadas del área de humanidades, correspondientes a los años 2006 y 2007, que han sido recogidos por la base de datos Arts and Humanities Citation Index $(\mathrm{A} \& \mathrm{HCl})$, contenida en el portal ISI Web of Knowledge. La estrategia de búsqueda ha consistido en la descarga de todos los documentos publicados en los años 2006 y 2007, contenidos en esta base de datos, a través de la consulta del campo fuente.

Los datos de cada referencia bibliográfica se han volcado en una base de datos gestionada en access, que nos ha permitido el tratamiento y análisis de los mismos.

\section{RESULTADOS}

Se han analizado un total de 10.445 referencias bibliográficas, contenidas en 559 documentos publicados en las cinco revistas seleccionadas durante los años 2006 y 2007.

\subsection{Producción científica}

En la tabla 1 se recoge la información sobre los trabajos publicados en las revistas analizadas durante el período de tiempo estudiado y su distribución por tipología documental. Como se puede observar, las revistas Arbor, Hispania y Revista de Indias, son las que han publicado un mayor número de documentos, tienen una mayor frecuencia de aparición y también 
son las revistas que tienen un mayor número de referencias bibliográficas.

Como ya hemos indicado anteriormente, las revistas de humanidades seleccionadas publicaron, durante los años 2006 y 2007 un total de 559 trabajos, en los cuales se incluyen un total de 10.445 citas bibliográficas a otros trabajos, cifra que supone un promedio de cerca de 19 referencias por trabajo. La distribución de las referencias bibliográficas por revistas, junto con el número total de documentos publicados y la media de referencias bibliográficas por trabajos se muestra en la tabla II. De las revistas estudiadas, la revista que tiene un mayor número de referencias por trabajo es la revista Al-Qantara, $(27,35)$, por el contrario, la revis- ta que presenta un menor número de referencias por trabajo es la revista Arbor $(12,89)$.

\subsection{Tipología documental}

La distribución de las referencias bibliográficas, atendiendo a la tipología de los documentos citados, se muestra en la figura 1 . Se puede observar que las citas bibliográficas son efectuadas, en su mayoría, a libros (67\%) y artículos de revistas (24\%). Estos dos tipos de documentos suponen el $91 \%$ del total de las referencias bibliográficas contenidas en los trabajos publicados en las cinco revistas analizadas durante el período estudiado. El resto de los tipos documentales (congresos, prensa, tesis) están escasamente representados.

Tabla I. Distribución de los documentos publicados por géneros documentales y años

\begin{tabular}{|l|c|c|c|c|c|c|c|c|c|c|c|}
\hline & \multicolumn{2}{|c|}{ Arbor } & \multicolumn{2}{c|}{ Al-Qantara } & \multicolumn{2}{c|}{ Dynamis } & \multicolumn{2}{c|}{ Hispania } & \multicolumn{2}{c|}{ Revista de indias } & \\
\hline Tipología documental & 2006 & 2007 & 2006 & 2007 & 2006 & 2007 & 2006 & 2007 & 2006 & 2007 & Total \\
\hline Article & 68 & 63 & 20 & 10 & 9 & 10 & 24 & 29 & 25 & 19 & 277 \\
\hline Bibliographical-Item & - & - & - & - & - & - & 1 & 1 & 1 & - & 3 \\
\hline Bibliography & 1 & - & - & - & - & - & - & - & - & - & 1 \\
\hline Book Review & 7 & 14 & 13 & 11 & 18 & 23 & 47 & 54 & 12 & 29 & 228 \\
\hline Editorial Material & 5 & 7 & - & 6 & - & 2 & - & 2 & 1 & 2 & 25 \\
\hline Fiction, Creative Prose & - & 1 & - & - & - & - & - & - & - & - & 1 \\
\hline Proceedings Paper & - & 1 & 1 & - & 2 & 3 & - & 1 & 1 & 5 & 14 \\
\hline Review & - & - & 1 & - & - & 1 & 4 & 2 & 2 & - & 10 \\
\hline Total & 81 & 86 & 35 & 27 & 29 & 39 & 76 & 89 & 42 & 55 & 559 \\
\hline
\end{tabular}

Tabla II. Distribución por revistas del número total de documentos publicados, referencias bibliográficas y media de referencias por documento

\begin{tabular}{|c|c|c|c|c|c|c|c|}
\hline \multirow{2}{*}{ Revistas } & \multicolumn{2}{|c|}{2006} & \multicolumn{2}{|c|}{2007} & \multirow{2}{*}{$\begin{array}{c}\text { Total } \\
\text { trabajos }\end{array}$} & \multirow{2}{*}{$\begin{array}{c}\text { Total } \\
\text { referencias }\end{array}$} & \multirow{2}{*}{$\begin{array}{c}\text { Referencias/ } \\
\text { trabajos }\end{array}$} \\
\hline & Trabajos & Referencias & Trabajos & Referencias & & & \\
\hline Al-Qantara & 35 & 1069 & 27 & 627 & 62 & 1696 & 27,35 \\
\hline Arbor & 81 & 923 & 86 & 1229 & 167 & 2152 & 12,89 \\
\hline Dynamis & 29 & 582 & 39 & 684 & 68 & 1266 & 18,62 \\
\hline Hispania & 76 & 1619 & 89 & 1725 & 165 & 3344 & 20,27 \\
\hline $\begin{array}{l}\text { Revista de } \\
\text { Indias }\end{array}$ & 42 & 1041 & 55 & 946 & 97 & 1987 & 20,48 \\
\hline Total & 228 & 4165 & 269 & 4584 & 559 & 10445 & 18,69 \\
\hline
\end{tabular}

Figura 1. Distribución de la totalidad de los trabajos citados según tipología documental

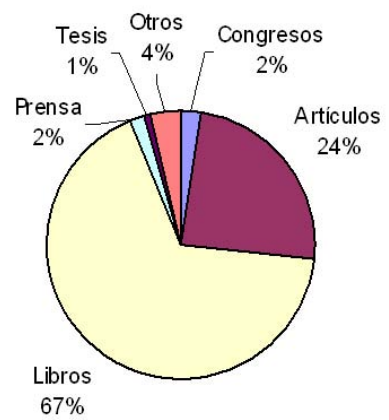


Sin embargo, el análisis pormenorizado de los distintos tipos documentales en cada una de las revistas analizadas, muestra que existen diferentes patrones de comportamiento en cada una de ellas, tal como se muestra en la figura 2. Como se puede observar las revistas Arbor (71,82\% de su producción total) y AlQantara (68,22\% de su producción total) son las que citan un mayor número de libros, mientras que Dynamis $(32,94 \%)$, Al-Qantara $(24,23 \%)$ son las revistas que citan un mayor número de artículos.

Respecto a los otros tipos de documentos, destacan las revistas Al-Qantara (4,07\%) e Hispania (3,20\%), por ser las revistas que citan un mayor número de actas de congresos, Hispania (2,99\%) y Arbor (2,57\%) por ser las revistas que más citan artículos de prensa y Revista de Indias (1,31\%) y Dynamis $(0,95 \%)$, por ser las que citan un mayor número de tesis doctorales.

Se observa que existe una gran dispersión en las revistas citadas. El número total de revistas citadas por las cinco revistas estudiadas, una vez eliminados los títulos duplicados por haber sido citadas en más de una revista, es de 1346, sin embargo, hay que indicar, tal como se muestra en la tabla III que, en la mayoría de los casos, abundan las revistas de las que solo se ha citado un trabajo.

Figura 2. Distribución en porcentajes de los trabajos citados en las revistas analizadas, según tipología documental

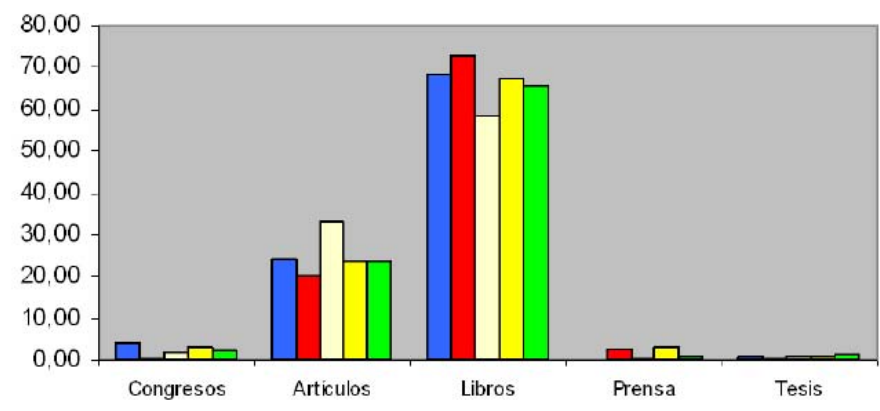

Revistas

๑Al-Qantara $\square$ Arbor $\square$ Dynamis $\square$ Hispania $\square$ Revista de Indias

Tabla III. Distribución por revistas del número total de documentos publicados, referencias bibliográficas y media de referencias por documento

\begin{tabular}{|c|c|c|c|c|c|c|c|c|c|}
\hline \multicolumn{2}{|c|}{ Al-Qantara } & \multicolumn{2}{|c|}{ Arbor } & \multicolumn{2}{|c|}{ Dynamis } & \multicolumn{2}{|c|}{ Hispania } & \multicolumn{2}{|c|}{ Revista de Indias } \\
\hline $\begin{array}{c}\text { No revistas } \\
\text { citadas }\end{array}$ & $\begin{array}{c}\text { № artículos/ } \\
\text { revista }\end{array}$ & $\begin{array}{l}\text { № revistas } \\
\text { citadas }\end{array}$ & $\begin{array}{c}\text { № artículos/ } \\
\text { revista }\end{array}$ & $\begin{array}{l}\text { № revistas } \\
\text { citadas }\end{array}$ & $\begin{array}{c}\text { № artículos/ } \\
\text { revista }\end{array}$ & $\begin{array}{l}\text { № revistas } \\
\text { citadas }\end{array}$ & $\begin{array}{c}\text { № artículos/ } \\
\text { revista }\end{array}$ & $\begin{array}{l}\text { № revistas } \\
\text { citadas }\end{array}$ & $\begin{array}{c}\text { Noo artículos/ } \\
\text { revista }\end{array}$ \\
\hline 1 & 45 & - & - & - & - & - & - & - & - \\
\hline 1 & 38 & - & - & - & - & 1 & 38 & - & - \\
\hline- & - & - & - & - & - & - & - & 1 & 26 \\
\hline- & - & - & - & 1 & 20 & - & - & - & - \\
\hline- & - & - & - & - & - & - & - & - & - \\
\hline- & - & - & - & 1 & 18 & - & - & - & - \\
\hline- & - & - & - & - & - & - & - & - & - \\
\hline- & - & - & - & 1 & 16 & - & - & 1 & 16 \\
\hline 1 & 15 & - & - & - & - & - & - & - & - \\
\hline 1 & 14 & - & - & - & - & - & - & - & - \\
\hline- & - & 1 & 13 & 1 & 13 & - & - & - & - \\
\hline- & - & - & - & - & - & 1 & 12 & 1 & 12 \\
\hline- & - & 1 & 11 & - & - & 1 & 11 & 1 & 11 \\
\hline 1 & 10 & - & - & 1 & 10 & - & - & 1 & 10 \\
\hline 2 & 9 & - & - & 1 & 9 & 1 & 9 & - & - \\
\hline- & - & 2 & 8 & 2 & 8 & - & - & 1 & 8 \\
\hline- & - & 1 & 7 & 3 & 7 & 7 & 7 & 3 & 7 \\
\hline 3 & 6 & 2 & 6 & - & - & 4 & 6 & 3 & 6 \\
\hline 3 & 5 & 4 & 5 & 3 & 5 & 8 & 5 & 1 & 5 \\
\hline 3 & 4 & 9 & 4 & 1 & 4 & 8 & 4 & 2 & 4 \\
\hline 13 & 3 & 11 & 3 & 9 & 3 & 28 & 3 & 18 & 3 \\
\hline 32 & 2 & 35 & 2 & 22 & 2 & 66 & 2 & 31 & 2 \\
\hline 120 & 1 & 217 & 1 & 202 & 1 & 363 & 1 & 223 & 1 \\
\hline 181 & & 283 & & 248 & & 488 & & 287 & \\
\hline
\end{tabular}


La relación de revistas más citadas durante los años 2006 y 2007, por las cinco revistas que hemos analizado, junto con el número de trabajos citados en cada una de ellas, y el país de edición de las mismas se muestra en la tabla IV. Se observa que en todas estas publicaciones, excepto en el caso de la revista Arbor, predominan las autocitas. También hay que destacar que, en general, las revistas más citadas están editadas en España (se da el caso, de que algunas de las revistas extranjeras más citadas, como es el caso de la revista Annales de l'Institut Pasteur, ha recibido todas sus citaciones de un mismo autor y un mismo trabajo).

Tabla IV. Ranking de las revistas más citadas por las revistas Al-Qantara, Arbor, Dynamis, Hispania y Revista de Indias, durante los años 2006 y 2007, junto el país de edición de la revista y número de citas recibidas

\begin{tabular}{|c|c|c|c|}
\hline \multicolumn{4}{|c|}{ Revistas más citadas por AL-QANTARA (2006-2007) } \\
\hline Rank & Revista & № citaciones & País \\
\hline 1 & AL-QANTARA & 45 & España \\
\hline 2 & AL-ANDALUS & 33 & España \\
\hline 3 & GACETA NUMISMATICA & 15 & España \\
\hline 4 & STUD ISLAMICA & 14 & Francia \\
\hline 5 & ARABICA & 9 & Holanda \\
\hline 5 & NUMISMA & 9 & España \\
\hline \multicolumn{4}{|c|}{ Revistas más citadas por ARBOR (2006-2007) } \\
\hline Rank & Revista & № citaciones & País \\
\hline 1 & GRAELLSIA & 13 & España \\
\hline 2 & INSULA & 11 & España \\
\hline 3 & ANN PHYS-BERLIN & 8 & Alemania \\
\hline 4 & QUIMERA & 8 & España \\
\hline 5 & NATURE & 7 & Reino Unido \\
\hline \multicolumn{4}{|c|}{ Revistas más citadas por DYNAMIS (2006-2007) } \\
\hline Rank & Revista & № citaciones & País \\
\hline 1 & DYNAMIS & 20 & España \\
\hline 2 & ANN I PASTEUR & 18 & Francia \\
\hline 3 & ASCLEPIO & 16 & España \\
\hline 4 & FARMACIA & 13 & España \\
\hline 5 & REV HYGIENE POLICE S & 10 & Francia \\
\hline \multicolumn{4}{|c|}{ Revistas más citadas por HISPANIA (2006-2007) } \\
\hline Rank & Revista & № citaciones & País \\
\hline 1 & HISPANIA & 38 & España \\
\hline 2 & AYER & 12 & España \\
\hline 3 & ANUARIO ESTUDIOS MED & 11 & España \\
\hline 4 & AL-QANTARA & 9 & España \\
\hline 5 & ANUARIO HIST DERECHO & 7 & España \\
\hline 5 & CHRONICA NOVA & 7 & España \\
\hline 5 & ESPANA MEDIEVAL & 7 & España \\
\hline 5 & HIST TEACHER & 7 & Estados Unidos \\
\hline 5 & RELATIONS INT & 7 & Francia \\
\hline 5 & REV ESTUDIOS POLITIC & 7 & España \\
\hline 5 & STUDIA HIST HIST MOD & 7 & España \\
\hline \multicolumn{4}{|c|}{ Revistas más citadas por REVISTA DE INDIAS (2006-2007) } \\
\hline Rank & Revista & № citaciones & Pais \\
\hline 1 & REV INDIAS & 26 & España \\
\hline 2 & ANUARIO ESTUDIOS AM & 16 & España \\
\hline 3 & REV FILOL ESPAN & 12 & España \\
\hline 4 & HISPANIC AM HIST REV & 11 & Estados Unidos \\
\hline 5 & TIERRA FIRME & 10 & Venezuela \\
\hline
\end{tabular}




\subsection{Redes de citas entre revistas}

Con el fin de medir la intensidad de las relaciones establecidas entre las cinco revistas analizadas, hemos aplicado la técnica de análisis de redes sociales al estudio de las mismas. Cuando dos o más trabajos citan una misma referencia, esta coincidencia puede ser interpretada como un indicador de la existencia de un vínculo entre los distintos trabajos. De la misma manera, cuanto más relacionadas están las revistas, más complementarios son sus artículos y más refleja la existencia de una disciplina coherente e integrada el agregado que forman (Callon, 1995). En este trabajo, para que se pueda ver con mayor claridad el gráfico de redes entre revistas citadas, se han representado en el mismo las revistas citadas conjuntamente, junto con una selección de solamente las revistas más citadas por cada una de las revistas estudiadas (ver figura 3). El tamaño de los nodos representa el peso de cada revista citada en la red (en este caso, hay que tener en cuenta que se están incluyendo también las autocitas) y el grosor de las líneas es un indicador de la intensidad de las relaciones entre las diferentes publicaciones.

Al observar el mapa podemos rápidamente diferenciar la existencia de dos subgrupos dentro de la red: un primer grupo formado por las revistas Arbor, Dynamis y Revistas de Indias, y un segundo grupo formado por las revistas Al-Qantara e Hispania. Destaca el papel que juegan tres revistas que actúan como enlace entre esos dos grupos, es decir, son las encargadas de unir o conectar a los dos grupos de revistas estudiadas, se trata de las revistas Asclepio, editada por el CSIC, dedicada a la publicación de trabajos de Historia de la Ciencia y de la Medicina (es citada por Arbor, Dynamis e Hispania), Ayer, revista de Historia, promo- vida por la Asociación de Historia Contemporánea y que esta dedicada al análisis de temas de actualidad (citada por Dynamis e Hispania) y Anuario de Estudios Americanos, editada por el CSIC, que publica trabajos sobre investigación histórica, aunque también publica trabajos de otras materias como la critica literaria, la ciencia política o la antropología (citada por Al-Qantara y Revista de Indias).

\section{DISCUSIÓN Y RESULTADOS}

Las referencias bibliográficas desempeñan varias funciones esenciales. Entre otras, sirven para reconocer las ideas y los hallazgos de otros investigadores, proporcionar información válida y útil a los lectores del artículo y, especialmente para refrendar afirmaciones y argumentos. La exactitud bibliográfica de cualquiera de las secciones que componen una revista especializada respalda la información vertida en ella por sus autores, ya que garantiza en gran medida que se han consultado las fuentes originales predecesoras.

En la referencia bibliográfica se realiza una descripción de los datos esenciales del material que se ha mencionado en el texto. Con ella se desea aludir a un antecedente bibliográfico relevante. Cada referencia bibliográfica debe ser veraz (completa y exacta) tanto en sus elementos formales de citación como en sus contenido (Ponce y Rodríguez, 1999). Para cumplir estos cometidos es natural que las referencias bibliográficas sean pertinentes $y$, además, exactas, ya que de otro modo sería difícil o imposible acceder a la fuente original (Pulido, 1997). Un aspecto crucial en la creación y diseminación de la información científica es el uso que hacen los autores en sus artículos de referencias bibliográficas a los trabajos de otros. Teóricamente, cuando

Figura 3. Red de revistas citadas

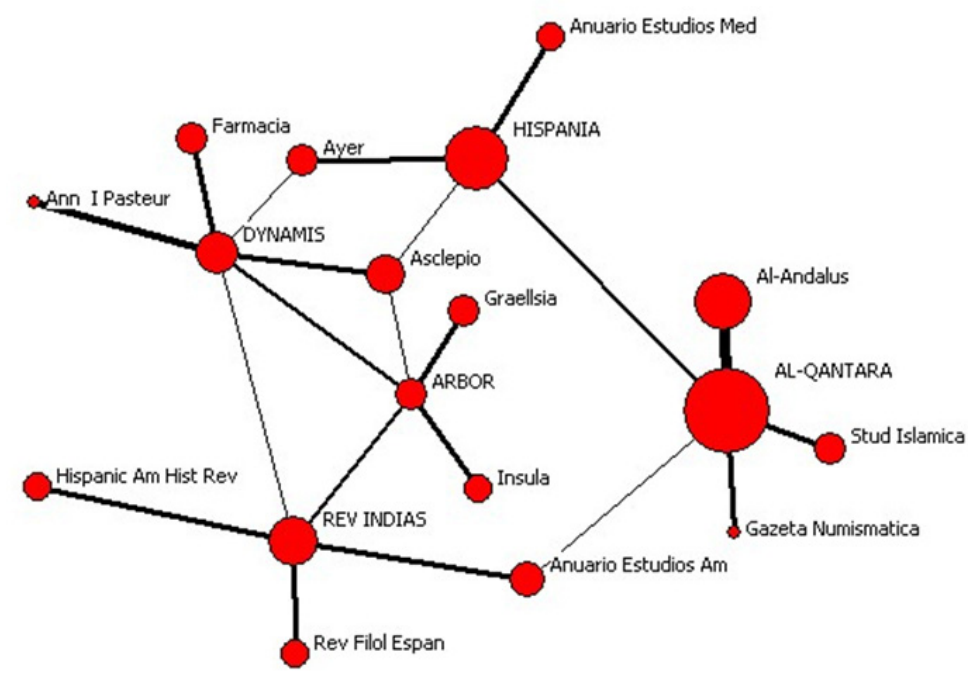


un autor cita a otro, está dando crédito a la tesis de su colega en un determinado trabajo, por lo que las citas representan las influencias que ha tenido un trabajo de los anteriores. Las citas y las referencias constituyen por tanto un aspecto importante en la comunicación científica. Es obvio que es necesario que el autor haya tenido acceso a los documentos para poder usarlos y por tanto citarlos. De esto se deduce que muchos trabajos que podrían ser citados no lo son por simple falta de acceso físico a ellos, y otros que el autor sí cita no son realmente relevantes, a pesar de que el autor los haya podido utilizar. De este modo, se tenderá a usar más aquellos documentos más próximos en términos de posibilidad de uso, y los grupos de autores que usen unas fuentes de documentación comunes tenderán a citar a las mismas fuentes, lo que generará enlaces bibliográficos entre ellos con origen en criterios de proximidad y no sólo de calidad. Una critica muy extendida a los análisis de citas deriva de la importancia que se ha dado a los mismos en ciertos ambientes, que ha originado un gran interés en ser citados por parte de los científicos. El uso indiscriminado de indicadores como el factor de impacto, calculado a partir del número de citas que recibe un trabajo, como medida de evaluación de los investigadores científicos, ha desembocado inevitablemente en una picaresca en la producción de referencias $y$, algunos estudios han demostrado que muchos autores simplemente copian las referencias de otros artículos para intentar reafirmar la consistencia de los propios (Broadus, 1983; Moed y Vriens, 1989).

Desde hace años, numerosos estudios publicados en la literatura corroboran el gran porcentaje de citas inexactas en artículos de diferentes especialidades cuando se escoge una muestra de referencias bibliográficas y se coteja con el documento original (Pulido, González y Sanz, 1995; Pulido, 1999; Ponce y Rodríguez, 1999). Las consecuencias de los errores en las referencias bibliográficas son de diversa índole. En primer lugar, en muchos casos, el error impide, en principio, localizar el documento original, sin embargo, en otro aspecto, el error en las referencia bibliográfica puede perjudica, a su vez, tanto a los autores, como a las propias revistas donde se han publicado los trabajos (Osca-Lluch et al., 2009), ya que algunos errores, traen como consecuencia, que las citaciones que reciben algunos de los trabajos publicados en revistas indizadas en bases de datos, como por ejemplo, las del ISI ( $\mathrm{AHCl}, \mathrm{SCl}$ y SSCl$)$ se pierdan. (no hay que olvidar que el número de citas que recibe un trabajo es un elemento fundamental para el calculo de algunos indicadores bibliométricos como el factor de impacto o el índice de Hirsch).

El análisis de citas y referencias también se utiliza para estudiar el consumo de información por parte de autores, grupos de trabajo, centros, revistas y países y conocer la repercusión que su producción ha tenido en la comunidad científica. Este análisis puede ser útil tanto para los profesionales de la información, que dispondrán de instrumentos válidos para conocer los mecanismos de la investigación y la estructura y dinámica de los colectivos de investigadores que producen y utilizan dicha literatura, como para los propios productores, consumidores y usuarios en general de la información, que podrán conocer los patrones que rigen la generación de información sobre una determinada área de la ciencia.

Los resultados obtenidos en este tipo de estudios deben, muy habituales en las áreas de ciencias, pero menos frecuentes en las áreas de humanidades, por las dificultades que conlleva la normalización de datos, por ello deben interpretarse con cautela, debido tanto a las limitaciones de las propias bases de datos, como a los errores, falta de implementación de datos y falta de normalización de las referencias analizadas, cuyo formato varía considerablemente según las diferentes normas de presentación de manuscritos que recomiendan los editores de las revistas.

En los datos analizados llama la atención que en todas las revistas analizadas las citas se realizan fundamentalmente a libros (67\%), siendo los trabajos publicados en revistas los documentos citados en segundo lugar (24\%), aunque existen diferentes patrones de comportamiento en cada una de las cinco publicaciones analizadas. Destaca la revista Arbor, por ser la publicación que cita más libros $(71,82 \%$ del total de sus referencias) y Dynamis, por ser, de todas ellas, la revista que citas más trabajos publicados en revistas (32,94\% del total de sus referencias). Podríamos, de esta forma afirmar que, de las cinco revistas analizadas, la revista Arbor es la que tiene un comportamiento más propio del área de Humanidades, mientras que la revista Dynamis, es la que tiene un comportamiento más alejado al de humanidades y más cercano al de otras áreas científicas.

Un hecho que viene un poco a reforzar esta teoría es que de todos los trabajos publicados en las cinco revistas que hemos analizado, están recogidos en la base de datos $\mathrm{AHCl}$ (Arts and Humanities Citation Index), sin embargo se observa que algunos de los trabajos publicados por cuatro de estas revistas, Arbor, Dynamis, Hispania y Revista de Indias, también han sido incluidos en las base de datos SSCI (Socials Science Citation Index), mientras que solamente algunos de los publicados en la revista Dynamis han sido incluidos en la base de datos SCl (Science Citation Index).

Otro aspecto que llama mucho la atención es que al contrario de lo que ocurre en otros estudios similares, se observa que las publicaciones más citadas son las españolas y no las norteamericanas, lo que viene a demostrar que hay, por lo tanto, un marcado consumo de información española en esta área y se con- 
firma que en ella tienen un gran peso los estudios de temas de ámbito nacional y local.

Al aplicar la metodología del análisis de redes al estudio de las redes de citación entre revistas, con el fin de medir la intensidad de las relaciones establecidas entre las cinco revistas analizadas, se observa la existencia de un vínculo entre los distintos trabajos publicados en las mismas, existiendo una gran com- plementariedad de los mismos y la existencia de una disciplina coherente e integrada.

\section{AGRADECIMIENTOS}

Este trabajo ha sido financiado por el Ministerio de Ciencia e Innovación a través del proyecto HUM200613389-C03-03.

\section{BIBLIOGRAFÍA}

Broadus, R. N. (1971): "The Literature of the Social Science: A Survey of Citation Studies". International Social Science Journal, no 23, pp. 236-243.

Burton, R. E.; Kleber, R. N. (1960): "The half-life of some scientific and technical literatures". American Documentation, no 11, pp. 18-22.

Callon, M.; Courtial, J. P.; Penan H. (1995): Cienciometría. El estudio cuantitativo de la actividad científica: de la bibliometría a la vigilancia tecnológica. Gijón, Trea.

Garfield, E. (1979): Citation Indexing. Its Theory and Application in Science, Technology and Humanities. New York, Wiley.

Kaplan, N. (1965): "The Norms of Citation Behavior. Prolegomena to the Footnote". American Documentation, no 16, pp. 179-184.

López López P.; Tortosa Gil, F. (2002): Los métodos bibliométricos en psicología. En: Nuevas tecnologías de la información y documentación en psicología. Barcelona, Ariel.

López Piñero, J. M.; Terrada, M. L. (1992): "Los indicadores bibliométricos y la evaluación de la actividad médico-científica (III). Los indicadores de producción circulación y dispersión, consumo de información y repercusión". Medicina Clínica (Barc), no 98, pp. 384-388.

Lozano Guadalajara, J. V.; Sáez Gómez, J. M. (1999): Análisis del consumo de información en la revista Rehabilitación (Madr) (1990-1995). Rehabilitación, 33 (2), pp. 107-112.

Martín Moreno, C. (1999): Hábitos y necesidades de información de los científicos experimentales encuadrados en el área de las ciencias de la vida. Tesis doctoral. Getafe: Universidad Carlos III de Madrid.

Massó Avila, J. J.; Valera, J. E.; Medina, F.; Bernabéu Lledó, M. (2001): “Análisis de consumo de información en la revista Fisioterapia (1991-1999)". Fisioterapia, no 23 , pp. 154-159.

Merton, R. K. (1977): La estructura normativa de la ciencia. En: La sociología de la ciencia. Investigaciones teóricas y empíricas. Madrid, Alianza, pp. 355-368.

Merton, R. K. (1969): "Behavior Patterns of Scientists". American Schoolar, 38, 197225

Moravcsic, M. J.; Murugesan, P. (1975): "Some Results on the Function and Quality of Citations". Social Studies of Science, no 5, pp. 86-92.
Ortega Prieto, J.L. (2004): “Análisis del consumo de información de una revista electrónica: análisis de los ficheros Log de Cybermetrics". Revista Española de Documentación Científica, no 27, pp. 455-468.

Osca-Lluch, J.; Díaz Rojo, A.; Mínguez, O. (1999): "Análisis del consumo de información en los artículos españoles de terminología médica". Boletín de la Asociación Andaluza de Bibliotecarios, no 555, pp. 19-39.

Osca-Lluch, J.; Civera, C.; Peñaranda, M. (2009): "Consecuencias de los errores en las referencias bibliográficas. El caso de la revista Psicothema". Psicothema, 21 (2), pp. 305-308.

Terrada, M. L.; López Piñero, J. M.; OscaLluch, J.; Almero, A. (1993): El libro médico español en los años ochenta. Estudio bibliométrico. Valencia, IEDHC.

Tosete Herranz, F. (2002): “Midiendo la historia moderna: el impacto de la revista Hispania a través de las revistas universitarias de historia moderna españolas". Hispania, no 210, pp. 41-64. 